\title{
New Directions in the Physics of One-dimensional Electron Systems
}

\author{
Dario Ferraro $^{\mathrm{a}}$, Niccolò Traverso Ziani, and Maura Sassetti \\ Dipartimento di Fisica, Università degli Studi di Genova and SPIN-CNR, \\ Via Dodecaneso 33, Genova, Italy
}

Received 28 November 2019

Published online 14 February 2020

\begin{abstract}
This volume is devoted to the latest developments in the physics of electrons in one-dimensional condensed matter systems. Particular attention is devoted to spin-orbit coupling and its consequences, that range from topological edge and bound states to the identification of possible host systems for odd frequency superconductivity. The possibility of using the methods of one-dimensional physics to describe higher dimensional correlated system is also addressed. The non-equilibrium properties are then inspected under different points of view: Generating states on demand, using such states for caloritronics, mimicking non-equilibrium properties on one-dimensional systems with quantum circuits. Our hope is that this volume could be beneficial and entertaining to both specialists and non-specialists in field.
\end{abstract}

Particles confined in one dimension are expected to show intriguing properties, even at the classical level. As a first consequence of the reduced dimensionality of their phase space, in fact, they can be unambiguously ordered. Moreover, as soon as they interact among each other, exciting one particle implies the excitation of all the neighbouring ones, so that most excitations of one-dimensional systems are collective, that is, truly many-body in their nature. These peculiarities survive at the quantum level, where they have striking consequences. As an example, it is worth to mention that many models are characterized by an extensive amount of local or quasi-local conserved quantities, implying ergodicity breaking and enabling to conceive applications in quantum technology. A maybe even more surprising concept related to one-dimensional physics was formulated by Haldane in his celebrated conjecture: The low energy properties of any one-dimensional system, being it made of fermions, bosons, or spins, are well described by a free bosonic theory as long as the system is gapless. As far as electrons in solids are concerned, the conjecture and the corresponding Luttinger liquid theory allowed, since the beginning of the ' $80 \mathrm{~s}$, for the explanation of the spectral and the transport properties of quasi-one-dimensional systems, quantum wires, carbon nanotubes, and edge states of quantum Hall systems.

Recently, a rebirth of the interest in one-dimensional electronic systems, that are the object of this volume, took place mainly thanks to the new physical phenomena implied by spin-orbit coupling. The review opening the collection, by Gindikin et al. [1], is hence devoted to such interaction mechanism and in particular to pair spin-orbit interaction. The quest for heterostructures based on multiple coupled one-dimensional

a e-mail: ferraro@fisica.unige.it 
systems, superconductors, and magnetic materials resulted in further conceptual and technological developments. The study of quantum wire networks offers an intriguing possibility: Describing interacting higher dimensional systems as composed by onedimensional, and hence more intuitive and theoretically friendly, structures coupled to each other. The implications of this idea are reported by Meng [2].

Again substantiated by the high degree of control of hybrid structures composed of spin-orbit coupled materials and superconductors, one-dimensional systems are becoming a fundamental playground for understanding a very mysterious state of matter, with far reaching consequences in superconducting spintronics: odd frequency superconductivity. A review about the topic is provided by Cayao et al. [3].

Going to the focal point, the main reason for the renewed interest is that some peculiar one-dimensional electronic systems, such as proximitized spin-orbit coupled quantum wires and topological insulators, can host, at their terminal points, non abelian anyons. Such quasi-particles are called Majorana zero modes and are predicted to play a relevant role in topological quantum computation. The transport properties associated to Majorana fermions are thoroughly reviewed within this issue $[4,5]$, by Jonckheere et al. and Schuray et al. The properties of Majorana zero modes in the 'Kitaev tie' configuration are analysed by Maiellaro et al. [6]. In the presence of interactions, even more exotic quasiparticles, dubbed parafermions, can be engineered. Differently from Majorana fermions, parafermions could allow for universal quantum computations. In reference [7], by Schmidt, the main properties of parafermions are reviewed.

The non-equilibrium properties of one-dimensional systems have also been object of renewed interest. Hwang et al. [8] report on the possibility of exploiting onedimensional systems in the framework of caloritronics. Moskalets et al. [9] inspect the on demand injection of states composed by two electron, generalizing the single electron sources conventionally considered in electron quantum optics experiments. Finally, Anthore et al. [10], show how the universality of the Luttinger liquid physics can be investigated experimentally through the measurement of transport properties of tunable quantum circuits mimicking the physics of a local impurity in a onedimensional correlated Fermi systems in a controlled way.

For a better readability of the Issue, we have divided it into three parts: Models and Phases, containing the contributions in references [1-3], Bound States, where references [4-7] are collected, and Charge and Energy Transport, consisting in references [8-10].

\section{References}

1. A. Gindikin, V.A. Sablikov, Eur. Phys. J. Special Topics 229, 503 (2020)

2. T. Meng, Eur. Phys. J. Special Topics 229, 527 (2020)

3. J. Cayao, C. Triola, A. Black Schaffer, Eur. Phys. J. Special Topics 229, 545 (2020)

4. T. Jonckheere, J. Rech, L. Raymond, A. Zazunov, R. Egger, T. Martin, Eur. Phys. J. Special Topics 229, 577 (2020)

5. A. Schuray, D. Frombach, S. Park, P. Recher, Eur. Phys. J. Special Topics 229, 593 (2020)

6. A. Maiellaro, F. Romeo, R. Citro, Eur. Phys. J. Special Topics 229, 637 (2020)

7. T.L. Schmidt, Eur. Phys. J. Special Topics 229, 621

8. S.-Y. Hwang, B. Sothmann, Eur. Phys. J. Special Topics 229, 683 (2020)

9. M.V. Moskalets, J. Kotilahti, P. Burset, C. Flindt, Eur. Phys. J. Special Topics 229, $647(2020)$

10. A. Anthore, D.M. Kennes, E. Boulat, S. Andergassen, F. Pierre, Eur. Phys. J. Special Topics 229, 663 (2020) 